\title{
ESTIMATIVA DE ALTURAS GEOIDAIS PARA O ESTADO DE SÃO PAULO BASEADA EM REDES NEURAIS ARTIFICIAIS
}

\author{
Maurício Roberto Veronez ${ }^{1}$, Sérgio Florêncio de Souza², \\ Marcelo Tomio Matsuoka² e Alessandro Ott Reinhardt ${ }^{1}$ \\ Recebido em 17 fevereiro, 2009 / Aceito em 18 novembro, 2009 \\ Received on February 17, 2009 / Accepted on November 18, 2009
}

\begin{abstract}
The information of height provided by the GNSS (Global Navigation Satellite System) is purely geometrical, and in most engineering papers, the height must be referenced to the geoid. Provided we have a sufficient number of Bench Marks (BMs) with known horizontal and vertical coordinates, it is nearly always possible to adjust mathematical expressions that allow for the interpolation of geoidal heights. The aim of this paper is to evaluate the efficiency of Artificial Neural Network (ANN) in the process of predicting geoidal heights, having the State of São Paulo as the area of study. The information used is based on a set of 157 BMs, evenly distributed all across the State. The horizontal coordinates (latitude and longitude) and the vertical coordinates (geometrical, orthometrical and geoidal heights) of these BMs are known. From the 157 BMs, 115 were used for the training of RNA and 42 in the process of simulation to assess the efficiency of the model proposed. Efficiency is based in determining the discrepancies (error) between known geoidal heights and those which were obtained by the neural model. As a contribution to this research, we have compared the values simulated with the Earth Gravitational Model 2008 (EGM2008) and with the MAPGE02004 as well. In terms of results, the RNA produced a mean absolute error of $0.19 \mathrm{~m} \pm 0.14 \mathrm{~m}$ and a strong correlation $\left(R^{2}=0.9871\right)$ with the values taken as true. Statistically, the tests showed that there was no difference between known geoidal heights and those which were provided by the neural model for a level of significance of $5 \%$. When we compare these results with the EGM2008 and MAPGE02004, the RNA has an error reduction of 0.07 and $0.44 \mathrm{~m}$, respectively.
\end{abstract}

Keywords: GPS, MAPGE02004, EGM2008, artificial neural networks, geoidal height.

RESUMO. A informação da altitude fornecida pelo sistema GNSS (Global Navigation Satellite System) é puramente geométrica, e na maioria dos trabalhos de engenharia a altitude deve estar referenciada ao geóide. Com um número suficiente de Referências de nível (Rn’s) com coordenadas horizontais e verticais conhecidas, quase sempre, é possível ajustar-se, pelo Método dos Mínimos Quadrados, expressões matemáticas que permitem interpolar as alturas geoidais. 0 objetivo deste trabalho foi avaliar a eficiência das Redes Neurais Artificiais (RNAs) no processo de predição de alturas geoidais tendo como área de estudo 0 Estado de São Paulo. As informações utilizadas basearam-se em um conjunto de 157 Referências de nível (Rn's) distribuídas uniformemente em todo Estado. Para estas Rn's são conhecidas suas coordenadas horizontais (latitude e longitude) e verticais (altitudes geométrica e ortométrica e altura geoidal). Das 157 Rn's, 115 foram utilizadas para 0 treinamento da RNA e 42 no processo de simulação para avaliar a eficiência do modelo proposto. A eficiência baseou-se em determinar as discrepâncias (erro) entres as alturas geoidais conhecidas e as obtidas pelo modelo neural. Como contribuição da pesquisa comparou-se também os valores simulados com 0 Earth Gravitational Model 2008 (EGM2008) e também com o MAPGE02004. Em termos de resultados a RNA proporcionou um erro absoluto médio de $0,19 \mathrm{~m} \pm 0,14 \mathrm{~m}$ com uma forte correlação $\left(R^{2}=0,9871\right)$ com os valores tomados como verdadeiros. Estatisticamente os testes realizados mostraram que não houve diferença entre as médias das alturas geoidais conhecidas e as fornecidas pelo modelo neural para um nível de significância de 5\%. Comparando-se os resultados com o EGM2008 e MAPGE02004 a RNA proporcionou uma redução no erro de $0,07 \mathrm{~m}$ e $0,44 \mathrm{~m}$, respectivamente.

Palavras-chave: GPS, MAPGE02004, EGM2008, redes neurais artificiais, altura geoidal.

\footnotetext{
1 Universidade do Vale do Rio dos Sinos - UNISINOS, Laboratório de Sensoriamento Remoto e Cartografia Digital - LASERCA, Programa de Pós-Graduação em Geologia, Av. Unisinos, 950, 93022-000 São Leopoldo, RS, Brasil. Tel.: (51) 3591-1100, ramal 1739; Fax: (51) 3590-8162 - E-mail: veronez@unisinos.br

2Universidade Federal do Rio Grande do Sul - UFRGS, Instituto de Geociências, Departamento de Geodésia, Laboratório de Pesquisas em Geodésia (LAGE0), Av. Bento Gonçalves, 9500, 91501-970 Porto Alegre, RS, Brasil. Tel.: (51) 3308-7519; Fax: (51) 3308-7302 - E-mails: sergio.florencio@ufrgs.br; tomio.matsuoka@ufrgs.br
} 


\section{INTRODUÇÃo}

Redes Neurais Artificiais (RNAs) são agrupamentos de unidades de processamento (neurônios ou nós) estruturados e interconectados, cujo funcionamento é análogo a uma estrutura neural de organismos inteligentes (Müller \& Fill, 2003). As RNAs extraem seu poder computacional da sua estrutura maciçamente paralela distribuída e da sua habilidade de aprender/generalizar, tornando possível a resolução de problemas complexos nas mais diferentes áreas do conhecimento (Haykin, 2001).

0 funcionamento das RNAs é inspirado no cérebro humano (Haykin, 2001) e elas têm sido utilizadas com sucesso em várias áreas do conhecimento. Segundo Galvão et al. (1999), em função de sua estrutura não-linear as RNAs conseguem captar características mais complexas dos dados, o que nem sempre é possível com a utilização das técnicas estatísticas ou métodos determinísticos tradicionais.

Para Müller \& Fill (2003) a grande vantagem das RNAs sobre os métodos convencionais é que para elas não há necessidade do conhecimento da teoria intrínseca do problema e nem a necessidade de analisar relações que não são totalmente conhecidas entre as variáveis envolvidas na modelagem.

A forma do geóide está diretamente relacionada ao campo da gravidade da Terra. No entanto, o elipsóide é uma superfície matemática com a forma e dimensões próximas ao geóide e é utilizado nos levantamentos geodésicos como a superfície de referência adequada ao posicionamento geográfico. As superfícies do geóide e do elipsóide não são coincidentes nem paralelas e a separação entre as duas superfícies é denominada ondulação ou altura geoidal. Pontualmente é expressa como altura geoidal ou anomalia geoidal. Essa ondulação pode atingir até dezenas de metros. A inclinação relativa dessas superfícies, em casos extremos é da ordem de 1' (um minuto de arco) (Gemael, 1999).

A importância de modelar com exatidão o geóide tem aumentado nos últimos anos com o advento dos sistemas de posicionamento por satélite (Global Navigation Satellite System - GNSS). As altitudes dos pontos fornecidas pelos sistemas GNSS são elipsoidais e não ortométricas. Para converter altitudes elipsoidais em ortométricas a relação entre geóide e elipsóide deve ser conhecida.

Existem modelos complexos usados para determinar a superfície geoidal a partir de informações gravimétricas. Dentre esses modelos podemos citar a aplicação da integral de Stokes (Heiskanen \& Moritz, 1967), com o método de colocação por mínimos quadrados (Moritz, 1980) ou com a transformada de Fourier (Schwarz et al., 1989). Essas técnicas baseiam-se em relações funcionais e/ou estocásticas entre anomalias ar livre e ondulações geoidais.

Alguns autores realizaram estudos utilizando RNAs em modelagem geoidal (Seager et al., 1999; Tierra \& De Freitas, 2002; Maia, 2003; Miranda et al., 2007) onde todos encontraram resultados satisfatórios.

Seager et al. (1999) utilizando uma RNA do tipo backpropagation modelaram a ondulação geoidal em uma região na Austrália tendo como variáveis latitude, Iongitude e anomalia ar livre obtendo um erro médio de 0,166 m e máximo de 0,711 m.

Tierra \& De Freitas (2002) implementaram, via RNAs, um método de interpolação de anomalia ar livre em uma região do Equador e os resultados encontrados foram melhores do que a interpolação por krigagem.

0 mesmo trabalho foi desenvolvido por Miranda et al. (2007) em uma área de $3^{\circ} \times 3^{\circ}$ em torno do Datum Vertical Brasileiro na cidade de Imbituba no Estado de Santa Catarina. Os resultados encontrados na estimativa da anomalia ar livre foram semelhantes aos da interpolação por krigagem.

Maia (2003) desenvolveu um modelo neural para obter alturas geoidais com erros inferiores a $0,5 \mathrm{~m}$ em uma região do Estado de São Paulo limitado pelas latitudes $-19^{\circ} \mathrm{a}-26^{\circ}$ e longitudes $-54^{\circ}$ a $-44^{\circ}$, cujas variáveis foram latitude, Iongitude e anomalia ar livre do modelo EGM96 (Earth Gravitational Mode/ 1996).

Em outras áreas das Geociências as RNAs vêm sendo pesquisadas no sentido de modelar fenômenos complexos e que envolvem variáveis de difícil obtenção. Neste caso modelos neurais estão sendo desenvolvidos com sucesso envolvendo informações não tão complexas e de fácil obtenção.

Alguns trabalhos de aplicações de RNAs no sentido de envolver variáveis de fácil obtenção para solucionar problemas geralmente muito complicados de serem resolvidos com a matemática convencional podem ser citados. Na modelagem de evapotranspiração e temperatura de superfície (Yang et al., 1997; Atluri et al., 1999; George, 2001; Kumar et al., 2002; Veronez et al., 2006; Zanetti et al., 2008; Mao \& Shi, 2008). Na Geofísica em classificação litológica (Andrade, 1997; Siripitayananon et al., 2001; Bhatt, 2002; Da Cunha et al., 2003; Yang et al., 2004; Hsieh et al., 2005). Em ciências do solo, através do conceito de pedotransfer function, que consiste em modelar um problema com variáveis de fácil obtenção laboratorial, vem evoluindo com 0 uso de RNAs (Zacharias \& Wessolek, 2007; Parasuraman et al., 2007; Jana, 2007). Em Sistema de Posicionamento Global, Da Silva (2003) desenvolveu um modelo neural para estimar as portadoras $L_{1}$ e $L_{2}$ tendo como entrada na RNA informações das pseudodistâncias (códigos C/A e P) baseado 
nas estações GPS da RBMC (Rede Brasileira de Monitoramento Contínuo). As RNAs também têm sido aplicadas para estimar 0 valor do TEC ( Total Electron Content) usando dados de estações GPS de monitoramento contínuo (Hernández-Pajares et al., 1997; Leandro, 2004).

Especificamente na questão de modelagem de geóide, utilizando informações de fácil acesso, tem-se a pesquisa desenvolvida por Reinke et al. (2007). 0 trabalho foi desenvolvido na região metropolitana da cidade de São Paulo/SP e os dados utilizados no treinamento da RNA foram: entrada da rede as coordenadas UTM e na saída altura geoidal advinda do sistema MAPGE02004, que é um programa desenvolvido pelo Instituto Brasileiro de Geografia e Estatística (IBGE). A RNA desenvolvida foi testada em 21 vértices com alturas geoidais conhecidas e 0 erro médio quadrático obtido foi de $0,10 \mathrm{~m}$.

No que foi exposto em termos de aplicabilidade das RNAs em diferentes áreas das Geociências os objetivos deste trabalho foram:

- Avaliar a eficiência de uma RNA do tipo backpropagation no processo de predição de alturas geoidais tendo como área de estudo o Estado de São Paulo. As informações utilizadas basearam-se em um conjunto de 157 Referências de nível (Rn's) distribuídas uniformemente em todo Estado. Das 157 Rn's, 115 foram utilizadas para 0 treinamento da RNA e 42 no processo de simulação;

- Verificar se o modelo neural proposto proporciona alguma melhoria em relação ao EGM2008 e também ao MAPGE02004.

\section{MATERIAIS E MÉTODO}

\section{Altura geoidal para treinamento e simulação da rede neural}

A área de estudo foi o Estado de São Paulo conforme mostra a Figura 1, onde estão ilustradas as Rn's utilizadas no treinamento e na simulação da RNA cujo sistema de referência das mesmas é o SIRGAS (Sistema de Referência Geocêntrico para as Américas). As alturas geoidais fazem parte do banco de dados do IBGE com um desvio padrão médio de $\pm 0,10 \mathrm{~m}$ (Souza, 2002).

Para 0 treinamento e simulação foram utilizadas 115 e 42 Rn's, respectivamente, correspondendo a aproximadamente $75 \%$ e $25 \%$ do conjunto total de dados conforme sugerido por Haykin (2001).

As alturas geoidais das 42 Rn's simuladas por RNAs foram comparadas com as conhecidas possibilitando algumas inferências estatísticas importantes em um processo de validação.
0 mesmo processo comparativo supracitado foi realizado com o MAPGE02004 e EGM2008.

Segundo IBGE (2008) o Modelo de Ondulação Geoidal possui uma resolução de $10^{\prime}$ de arco baseado na integral modificada de Stokes, através da técnica de transformada rápida de Fourier (FFT). As informações utilizadas no processo foram:

- anomalias médias de Helmert em quadrículas de $10^{\prime} \times 10^{\prime}$ em áreas continentais, obtidas a partir de informações gravimétricas do IBGE, e de diversas instituições no Brasil e em países vizinhos;

- anomalias ar livre derivadas da altimetria por radar a bordo de satélite em áreas oceânicas, utilizando o modelo KMS99;

- modelo digital de terreno com resolução de $1^{\prime} \times 1^{\prime}$ obtido a partir da digitalização de cartas topográficas e, quando não disponíveis, utilizando o modelo GLOBE;

- modelo de geopotencial EGM96 até grau e ordem 180".

Na Assembléia Geral da União Européia de Geociências, realizada de 13 a 18 de abril de 2008 em Viena na Áustria, foi divulgado 0 novo EGM2008. 0 modelo é completo até 0 grau e ordem 2159, e contém coeficientes adicionais dos harmônicos esféricos até 0 grau 2190 e ordem 2159, 0 que resulta em um arquivo com 2.401.333 de coeficientes (Pavlis et al., 2008). Apesar do EGM2008 apresentar uma resolução muito superior aos antecessores, como no caso do EGM96, ainda não se pode afirmar a eficiência desse modelo na América do Sul. Assim para o correto uso desse modelo, sobretudo no Brasil, é necessária a sua avaliação. Sendo que, qualquer elemento do campo de gravidade pode ser utilizado nessa análise.

\section{Estrutura da rede neural utilizada}

Utilizou-se uma RNA do tipo perceptron de múltiplas camadas (MLP, multilayer perceptron) onde o princípio deste algoritmo baseia-se no aprendizado por correção de erro. Quando um padrão é apresentado à rede pela primeira vez, essa produz uma saída aleatória. A diferença entre essa saída e a desejada constitui 0 erro, que é calculado pelo próprio algoritmo. 0 algoritmo backpropagation faz com que os pesos da camada de saída sejam os primeiros a serem ajustados e, posteriormente, os pesos das restantes camadas, corrigindo-os de trás para frente, com o objetivo de reduzir o erro. Este processo é repetido durante 0 aprendizado até que 0 erro se torne aceitável (Silva et al., 2004). 


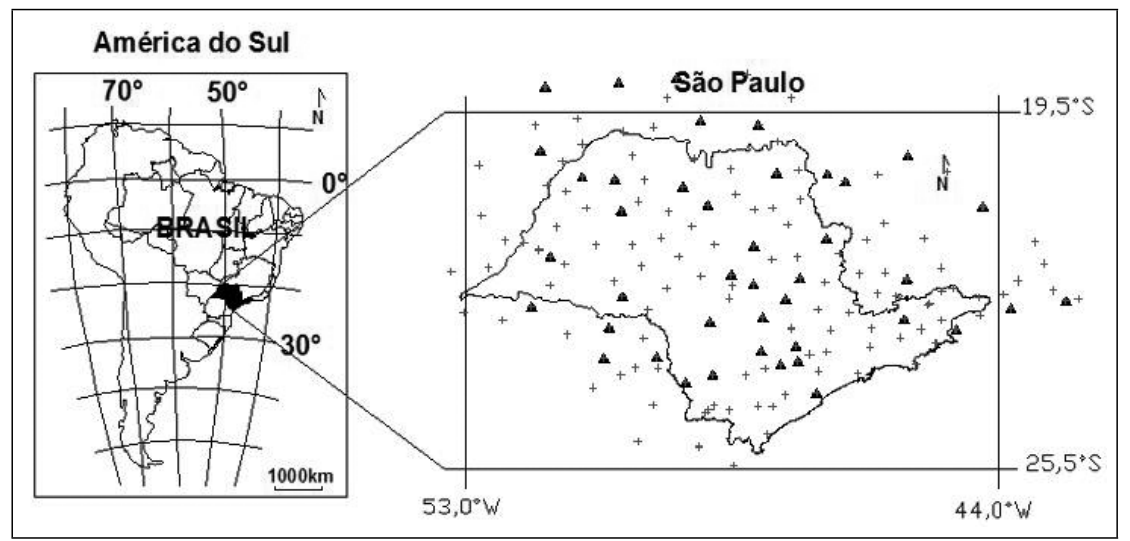

Figura 1 - Localização da área de estudo e distribuição das Rn's utilizadas no treinamento (+) e na simulação da Rede Neural ( $\mathbf{\Lambda}$ )

Os neurônios presentes na RNA utilizada foram configurados com base no modelo apresentado por Haykin (2001) conforme demonstra a Figura 2. Nos pesos sinápticos $\left(w_{k, j}\right) 0$ índice $k$ se refere ao neurônio em questão, enquanto 0 índice $j$ diz respeito ao sinal de entrada da sinapse com a qual o peso tem relação. A função do peso é multiplicar o sinal na entrada da sinapse conectada ao neurônio. As RNAs podem apresentar também pesos adicionais, denominados "bias", que tem a função de evitar a geração de erros quando todos os dados de entrada são nulos, pois assim, a matriz de pesos não sofre modificações no treinamento. A função de ativação é uma função de ordem interna, sendo uma decisão tomada pelo próprio neurônio sobre o que fazer com o valor resultante do somatório das entradas ponderadas. A função de transferência é uma função de saída ou limiar lógico. Ela controla a intensidade de ativação para se obter o desempenho desejado na rede.

Matematicamente a estrutura da Figura 2 pode ser expressa nas Equações 1, 2 e 3.

$$
\begin{gathered}
u_{k}=\sum_{j=1}^{n}\left(w_{k, j} \cdot x_{j}\right) \\
v_{k}=u_{k}+b_{k} \\
y_{k}=\varphi\left(v_{k}\right)
\end{gathered}
$$

onde:

- $u_{k}$ é a saída do combinador linear (junção aditiva);

- $w_{k, j}$ são os pesos sinápticos;

- $x_{j}$ são as variáveis de entrada;

- $v_{k}$ é o potencial de ativação;
- $b$ é o bias;

- $y_{k}$ é 0 sinal de saída do neurônio $k$;

- $\varphi\left(v_{k}\right)$ é a função de ativação.

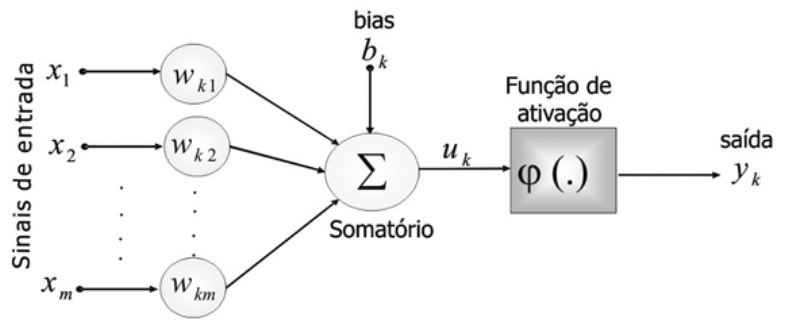

Figura 2 - Estrutura dos neurônios artificiais utilizados na RNA. Adaptada de Haykin (2001).

Utilizou-se um treinamento supervisionado da rede através do algoritmo de Levenberg-Marquardt, o qual utiliza o método de Newton, para aproximação do mínimo da função de erro (Haykin, 2001). Neste caso a RNA é treinada através da apresentação de pares de entradas e saídas, ou seja, para cada entrada fornecida para a rede, existe uma saída esperada que também é fornecida para 0 treinamento. A rede produz uma resposta na saída onde a mesma é comparada com a saída desejada (que foi fornecida). A diferença entre a resposta da rede e a resposta desejada (conhecida), gera um resíduo (erro). Esse erro obtido é utilizado, então, para calcular o ajuste necessário aos pesos sinápticos da rede, que serão corrigidos até que a resposta da rede coincida com a saída desejada. Esse é o processo de minimização de erro (Haykin, 2001).

Ainda (Haykin, 2001), nesse tipo de aprendizado, os cálculos necessários para minimizar 0 erro são importantes e estão atrelados ao algoritmo utilizado, como no backpropagation, por exemplo, onde são considerados parâmetros como: número de 
interações por padrão de entrada para 0 erro alcançar um valor mínimo no treinamento, a ocorrência de um mínimo local ou global e a capacidade da rede escapar dos mínimos locais.

A Equação 4 mostra a função de erro MSE (Mean Squared Error) a ser minimizada na fase de treinamento:

$$
M S E=\frac{\sum_{j=1}^{n}\left(d_{j}-y_{j}\right)^{2}}{n}
$$

onde:

- $d_{j}$ valor de saída desejado da RNA;

- $y_{j}$ valor de saída obtido.

Com o objetivo de selecionar uma RNA que proporcionasse 0 melhor desempenho, foram realizados diversos testes, modificando o número de camadas intermediárias, o número de neurônios por camada e a função de ativação possibilitando a escolha da melhor RNA para estimar a altura geoidal.

As variáveis das camadas de entrada e saída da RNA foram normalizadas no intervalo [0-1].

\section{Análises dos resultados}

A análise estatística utilizada na apresentação dos resultados da pesquisa baseou-se em comparar os valores de alturas geoidais modelados via RNA e os tomadas como verdadeiros. Foram utilizados o teste estatístico " $t$-Student" e a análise do coeficiente de determinação $R^{2}$ da regressão linear.

- Seja $x$ a medida de certo atributo dos elementos de uma população A (ondulação modelada por RNA);

- Seja y a medida do mesmo atributo dos elementos de uma população B (ondulação conhecida);

- Sejam $x$ e $y$ normalmente distribuídos com variâncias desconhecidas;

- Seja a hipótese: $\mu_{x}=\mu_{y}$ em que $\mu_{x}=$ média de $x$ e $\mu_{y}=$ média de $y$.

Para testar a hipótese da igualdade das médias das duas populações utilizou-se 0 teste $t$. Para isso foi necessário testar inicialmente se as duas populações apresentavam variâncias iguais utilizando-se 0 teste $\mathrm{F}$ de Fisher:

$$
\begin{gathered}
F=\frac{S Q D_{x}}{S Q D_{y}} \\
s_{x}^{2}=\frac{S Q D_{x}}{\left(n_{x}-1\right)} \\
s_{y}^{2}=\frac{S Q D_{y}}{\left(n_{y}-1\right)}
\end{gathered}
$$

$$
F_{\text {calculado }}=\frac{s_{x}^{2}}{s_{y}^{2}}
$$

onde:

- $S Q D_{x}$ e $S Q D_{y}$ correspondem, respectivamente, às somas dos quadrados dos desvios de $x$ e $y$;

- $s_{x}^{2}$ e $s_{y}^{2}$ correspondem, respectivamente, às variâncias amostrais de $x$ e $y$;

- $n_{x}$ e $n_{y}$ correspondem, respectivamente, ao número de variáveis de $x$ e $y$.

A hipótese testada $\left(H_{0}\right)$ foi que a variância populacional de $x$ é igual à variância populacional de $y$. Se $\operatorname{Prob}>F$ for menor que 5\% aceita-se $H_{0}$. Se Prob $>F$ for maior que $5 \%$ rejeita-se $H_{0}$. Se as variâncias populacionais forem estatisticamente iguais, calcula-se uma variância comum $\left(s_{c}^{2}\right)$ :

$$
\begin{gathered}
s_{c}^{2}=\frac{\left(S Q D_{x}+S Q D_{y}\right)}{\left[\left(n_{x}-1\right)+\left(n_{y}-1\right)\right]} \\
s_{c}^{2}=\frac{\left[\left(s_{x}^{2} \cdot\left(n_{x}-1\right)\right)+\left(s_{y}^{2} \cdot\left(n_{y}-1\right)\right)\right]}{\left[\left(n_{x}-1\right)+\left(n_{y}-1\right)\right]}
\end{gathered}
$$

A seguir testou-se $H_{0}$ para a igualdade das médias populacionais utilizando-se a variável aleatória $t$, definida por:

$$
t=\frac{\mu_{x}-\mu_{y}}{\sqrt{v \cdot\left(\mu_{x}-\mu_{y}\right)}}
$$

sendo:

$$
v \cdot\left(\mu_{x}-\mu_{y}\right)=v\left(\mu_{x}\right)+v\left(\mu_{y}\right)=\frac{s_{x}^{2}}{n_{x}}+\frac{s^{2} y}{n_{y}}
$$

onde:

- $v$ corresponde à variância da média. 
Admitindo $s_{x}^{2}=s_{y}^{2}=s_{c}^{2}$ tem-se:

$$
\begin{gathered}
v \cdot\left(\mu_{x}-\mu_{y}\right)=\frac{s_{c}^{2}}{n_{x}}+\frac{s_{y}^{2}}{n_{y}}=s_{c}^{2} \cdot\left(\frac{1}{n_{x}}+\frac{1}{n_{x}}\right) \\
t=\frac{\mu_{x}-\mu_{y}}{\sqrt{s_{c}^{2} \cdot\left(\frac{1}{n_{x}}+\frac{1}{n_{y}}\right)}}
\end{gathered}
$$

graus de liberdade $(n)=\left(n_{x}+n_{y}-1\right)$

No caso de variâncias diferentes tem-se:

$$
t=\frac{\mu_{x}-\mu_{y}}{\sqrt{\frac{s_{x}^{2}}{n_{x}}+\frac{s_{y}^{2}}{n_{y}}}}
$$

Sendo o grau de liberdade calculado com a seguinte equação:

$$
n=\frac{\left(\frac{s_{x}^{2}}{n_{x}}+\frac{s_{y}^{2}}{n_{y}}\right)^{2}}{\frac{\left(\frac{s_{x}^{2}}{n_{x}}\right)^{2}}{n_{x}-1}+\frac{\left(\frac{s_{y}^{2}}{n_{y}}\right)^{2}}{n_{y}-1}}
$$

\section{RESULTADOS E DISCUSSÕES}

A RNA que apresentou o melhor desempenho foi constituída com uma camada de entrada (2 variáveis), duas camadas sendo a primeira com 5 neurônios e a segunda com 4 neurônios e uma camada de saída (com 1 neurônio) conforme a Figura 3. 0 fato da rede escolhida, que apresentou melhor desempenho, ter mais de uma camada intermediária, está de acordo com os resultados encontrados por Maia (2003) e Reinke et al. (2007), pois esses autores modelaram altura geoidal com apenas 2 variáveis de entrada e concluíram que uma RNA com apenas uma camada intermediária não é suficiente para representar a relação não-linear entre informações posicionais e a variável modelada.
A função de ativação utilizada foi a sigmoidal logística:

$$
\varphi(v)=\frac{2}{1+\exp (-2 v)}-1
$$

0 número de ciclos de treinamento foi de 600. Na Figura 4 têm-se os valores de alturas geoidais conhecidas (máximo de $-0,36 \mathrm{~m}$, mínimo de $-8,77 \mathrm{~m}$ e médio de $-5,08 \mathrm{~m}$ ) e modeladas por RNAs (máximo de -0,36 m, mínimo de -8,92 e médio de $-5,05 \mathrm{~m}$ ) onde é possível verificar um comportamento semelhante entre as duas curvas. Verificaram-se discrepâncias (erros) máxima e mínima de 0,41 m e -0,80, respectivamente, com um comportamento semelhante entre as duas curvas. Em termos absolutos, a discrepância média foi de $0,19 \mathrm{~m}$ com um desvio padrão de $\pm 0,14 \mathrm{~m}$.

Já na Figura 5 tem-se um comparativo de valores de alturas geoidais conhecidas com as obtidas pelo EGM2008. As discrepâncias máxima e mínima foram de 0,29 m e -1,64, respectivamente, e também com um comportamento semelhante entre as duas curvas. Em termos absolutos a discrepância média foi de 0,26 m com um desvio padrão de $\pm 0,27 \mathrm{~m}$.

Comparando os resultados simulados pelo modelo EGM2008, as RNAS proporcionaram em média, uma melhora de $0,07 \mathrm{~m}$ no processo de interpolação de alturas geoidais dando indícios da eficiência do método proposto.

Se analisarmos as alturas geoidais obtidas pelo MAPGE02004, as discrepâncias máxima e mínima encontradas foram de $1,43 \mathrm{me}-0,66$, respectivamente. Em valores absolutos a discrepância média obtida foi de 0,63 m com um desvio padrão de $\pm 0,34 \mathrm{~m}$. Avaliando os resultados simulados o modelo neural também se mostrou mais eficiente na obtenção da altura geoidal. A melhora, comparativamente com o MAPGEO, foi significativa com uma redução do erro de aproximadamente 3 vezes.

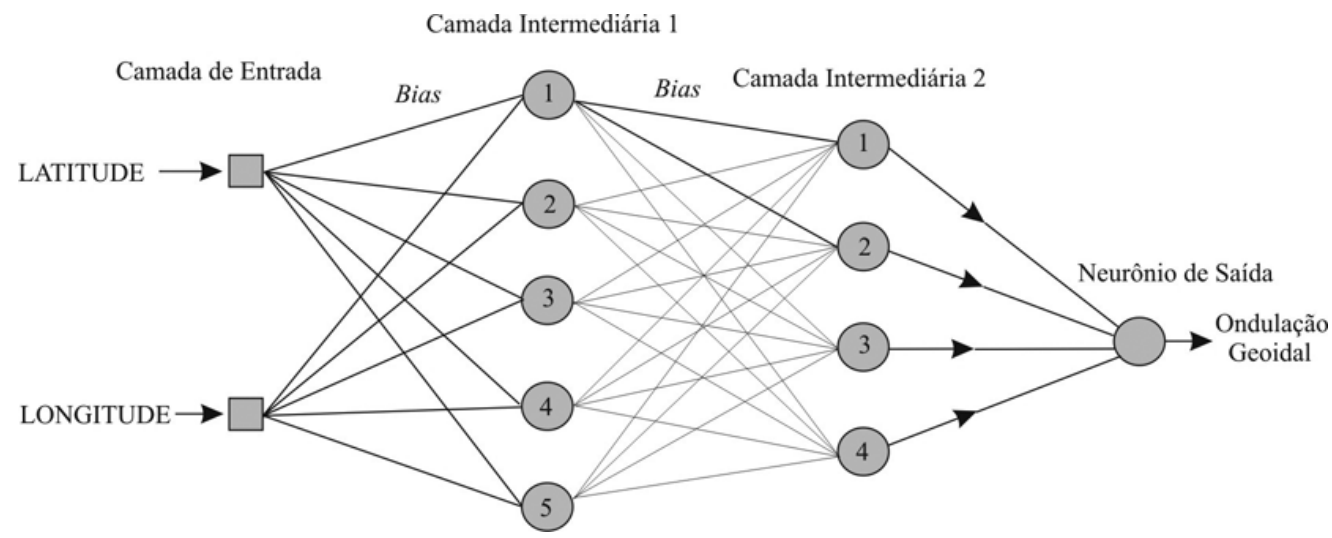

Figura 3 - Estrutura da rede neural utilizada na modelagem da altura geoidal. 


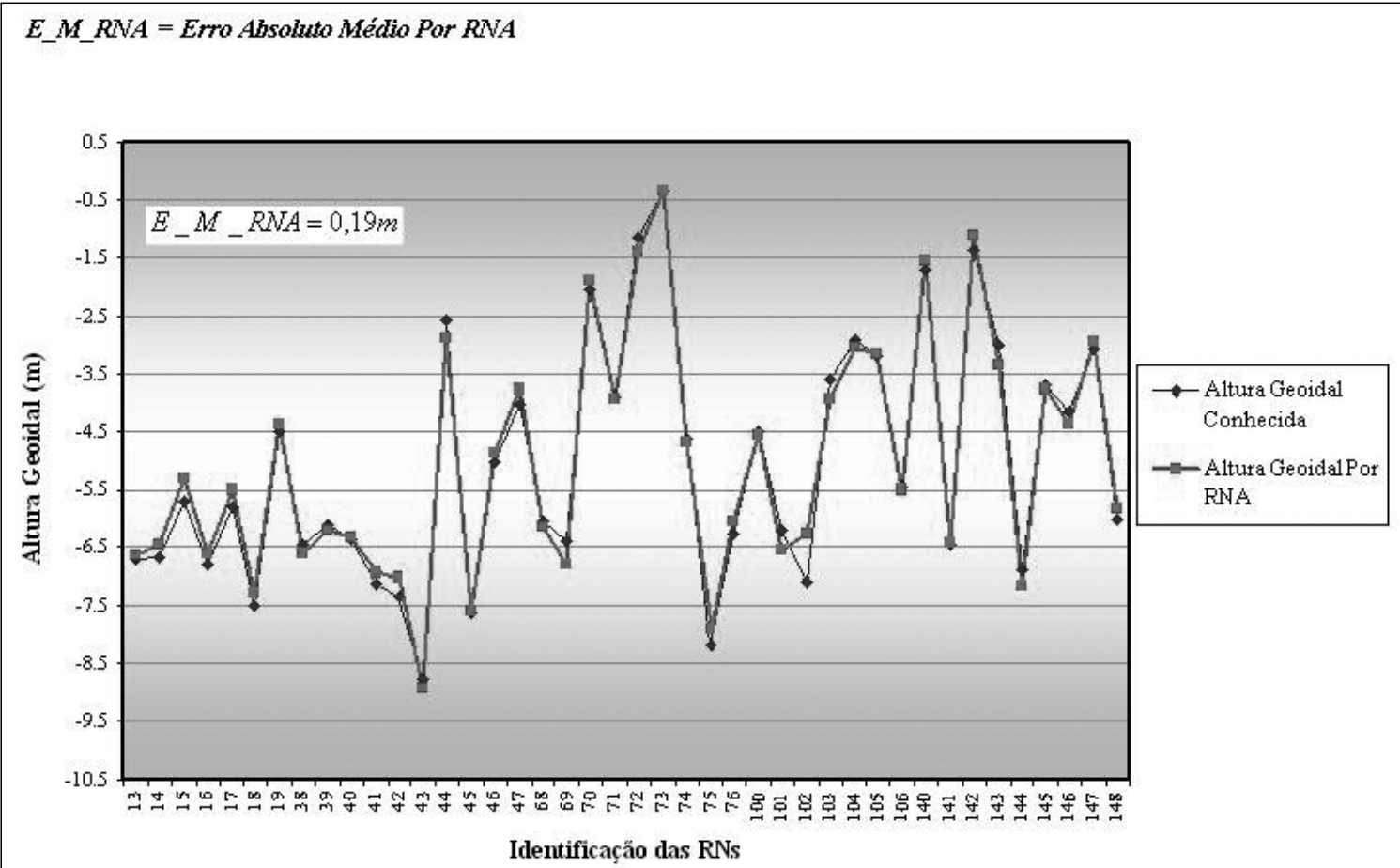

Figura 4 - Alturas geoidais conhecidas e obtidas por RNAs.

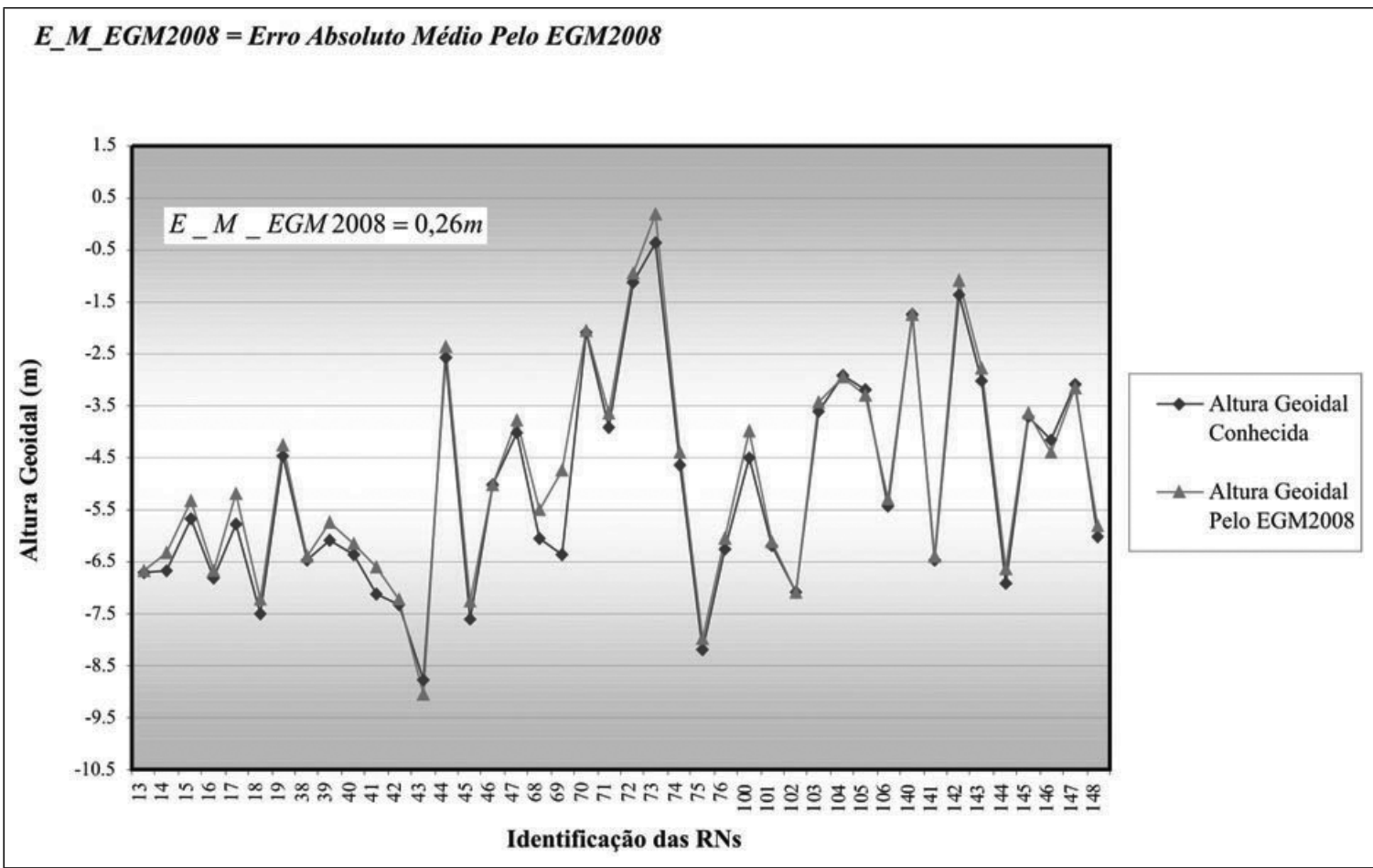

Figura 5 - Alturas geoidais conhecidas e obtidas pelo EGM2008. 


\section{E_M_MAPGEO= Erro Absoluto Médio Pelo MAPGEO2004}

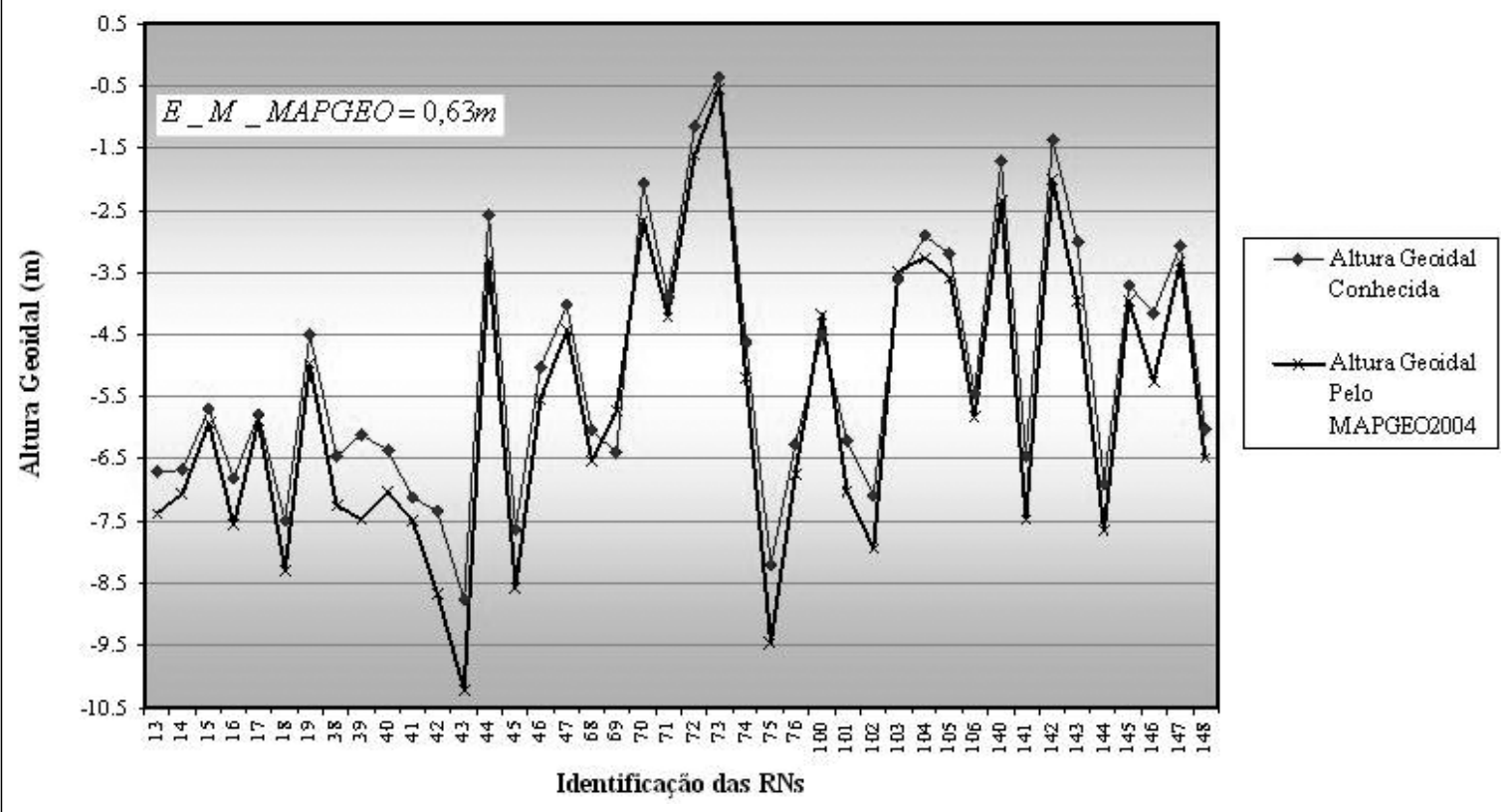

Figura 6 - Alturas geoidais conhecidas e obtidas pelo MAPGE02004.

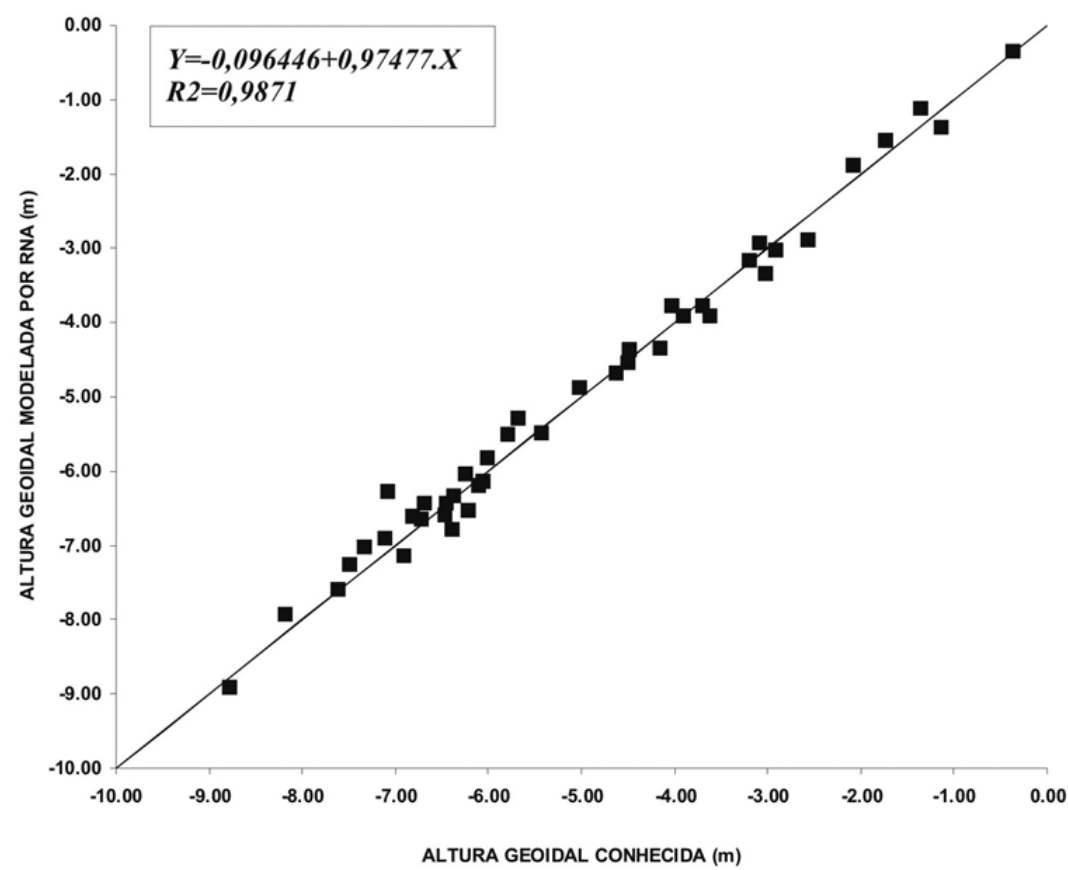

Figura 7 - Regressão linear entre valores de alturas geoidais conhecidas e modeladas por RNA.

Para avaliar a eficiência do modelo neural realizou-se uma análise de regressão (Fig. 7) onde verificou-se uma forte correlação entre os valores modelado e conhecido $\left(R^{2}=0,9871\right)$ dando indícios da eficiência da RNA proposta no processo de interpolação de altura geoidal.

Além da análise de regressão efetuou-se um teste de hipótese para analisar se 0 modelo proposto é estatisticamente igual ao tomado como verdadeiro. 
Tabela 1 - Indicadores estatísticos entre os valores de alturas geoidais modeladas por RNAs e as tomadas como verdadeiros $\left(\mathrm{M}_{1}=\right.$ valores verdadeiros e $\mathrm{M}_{2}=$ valores simulados $)$.

\begin{tabular}{|c|c|c|c|c|c|c|c|}
\hline M & N & Média $(\mathrm{m})$ & Desvio Padrão $(\mathrm{m})$ & Variância & $t$ & $\begin{array}{c}\text { Grau de } \\
\text { de liberdade }\end{array}$ & Prob $>(t)$ \\
\hline 1 & 42 & $-5,08$ & 2,65 & Igual & $-0,07$ & 82 & 0,944 \\
\hline 2 & 42 & $-5,05$ & 2,60 & Desigual & $-0,07$ & 82 & 0,944 \\
\hline \multicolumn{5}{l}{ ara $H_{0}$ : variâncias são iguais, $F^{\prime}=1,04 ; \quad D F=(41,41) ;$ Prob $>F^{\prime}=0,904$} \\
\hline
\end{tabular}

Para um nível de significância de $5 \%$, através do teste " $t$ Student" avaliou-se a igualdade das duas médias $\left(\mathrm{M}_{1}\right.$ e $\left.\mathrm{M}_{2}\right)$. A hipótese testada foi $\mathrm{M}_{1}=\mathrm{M}_{2}$. Nesse caso se Prob $>t$ fosse menor que 0.05 rejeitar-se-ia a hipótese e então $\mathrm{M}_{1}$ seria diferente de $\mathrm{M}_{2}$. No caso, os valores de alturas geoidais simuladas por RNAs e as conhecidas apresentaram valores médios estatisticamente iguais. A Tabela 1 mostra os resultados do teste estatístico realizado.

\section{CONCLUSÕES}

0 trabalho propôs um método de predição de valores de alturas geoidais para 0 Estado de São Paulo baseado em uma RNA treinada de forma supervisionada. Para isso foram utilizadas 157 Rn's distribuídas uniformemente em todo o Estado. Deste conjunto, 115 foram utilizados para 0 treinamento da rede neural e 42 para simulação. As variáveis envolvidas no modelo foram informações posicionais como entrada da rede (Latitude e Longitude) e como saída a altura geoidal.

No processo de simulação os resultados obtidos por RNAs mostraram uma melhora de $0,07 \mathrm{~m} \mathrm{e} 0,44 \mathrm{~m}$, respectivamente, se comparados com EGM2008 e o MAPGE02004.

Estatisticamente verificou-se que o modelo neural é fortemente correlacionado comparado ao tomado como verdadeiro $\left(R^{2}=0,9871\right)$ e que para um nível de significância de $5 \%$ não existe diferença entre ambos.

A grande vantagem observada do modelo neural em relação ao EMG2008 e ao MAPGE02004 é a quantidade mínima de pontos no processo de treinamento com a manutenção da estabilidade da solução o que pode ser observado pela melhora nos resultados.

Mesmo os testes terem indicados valores médios de alturas geoidais (simuladas e conhecidas) estatisticamente iguais, não se pode concluir que o modelo proposto pode ser aplicado em qualquer região brasileira. É um estudo preliminar e novos experimentos estão sendo realizados no sentido de melhorar a ava- liação da eficiência da RNA no processo de determinação de altura geoidal. Para isso novos modelos neurais estão sendo avaliados, densificando a malha de pontos nos processos de treinamento e simulação da rede, testando algoritmos diferentes do utilizado neste trabalho bem como as funções de ativação e ciclos de treinamento.

\section{REFERÊNCIAS}

ANDRADE AJN. 1997. Aplicação de redes neuronais artificiais na interpretação de perfis de poço aberto. Tese de Doutorado, Curso de PósGraduação em Geofísica, Universidade Federal do Pará, Belém. 127 p.

ATLURI V, HUNG CC \& COLEMAN TL. 1999. Artificial Neural Network for Classifying and Predicting Soil Moisture and Temperature Using Levenberg-Marquardt Algorithm. Alabama, p. 10-13.

BHATT A. 2002. Reservoir properties from well logs using neural networks. Doctor Thesis Department of Petroleum Engineering and Applied Geophysics, Norwegian University of Science and Technology. $157 \mathrm{p}$.

DA CUNHA ES, OLIVEIRA KA \& GOMES HM. 2003. Investigação do treinamento de uma rede neural para o reconhecimento de litofácies combinando dados de testemunhos e perfis de poços de petróleo. In: Congresso Brasileiro de P\&D em Petróleo \& Gás, 2., Rio de Janeiro. Anais... Rio de Janeiro, 2003. Artigos, p. 1-6, Online. Disponível em: <http://www.portalabpg.org.br/PDPetro/2/1022.pdf>. Acesso em: 11 set. 2008.

DA SILVA CAU. 2003. Um método para estimar observáveis GPS usando redes neurais artificiais. Escola de Engenharia de São Carlos - Universidade de São Paulo, Tese de Doutorado, 2003, 113 p.

GALVÃO CO, VALENÇA MJS, VIEIRA VPPB, DINIZ LS, LACERDA EGM, CARVALHO ACPLF \& LUDERMIR TB. 1999. Sistemas inteligentes: Aplicações a recursos hídricos e ciências ambientais. Porto Alegre: UFRGS/ABRH, $246 \mathrm{p}$.

GEMAEL C. 1999. Introdução à Geodésia Física. Editora da Universidade Federal do Paraná, Curitiba, 304 p. 
GEORGE RK. 2001. Prediction of soil temperature by using artificial neural networks algorithms. Nonlinear Analysis, 47(3): 1737-1748.

HAYKIN S. 2001. Redes Neurais: princípios e prática. Porto Alegre: Editora Bookman, $900 \mathrm{p}$.

HEISKANEN WA \& MORITZ H. 1967. Physical Geodesy. W.H. Freeman and Company, San Francisco, 364 p.

HERNÁNDEZ-PAJARES M, JUAN JM \& SANZ J. 1997. Neural network modeling of the ionospheric electron content at global scale using GPS data. Radio Science, 32(3): 1081-1089.

HSIEH B, LEWIS C \& LIN Z. 2005. Lithology of aquifers from geophysical well logs and fussy logic analysis: Shui-Lin Area, Taiwan. Computers \& Geosciences, 31(3): 263-275.

IBGE - Instituto Brasileiro de Geografia e Estatística. 2008. Geociências. Disponível em: <http://www.ibge.gov.br/home/geociencias/geodesia/ modelo_geoidal.htm> Acesso em: 2 set. 2008.

JANA RB. 2007. Multiscale pedotransfer functions for soil water retention. Vadose Zone Journal, 6(4): 868-878.

KUMAR M, RAGHUWANSHI NS, SINGH R, WALLENDER WW \& PRUITT WO. 2002. Estimating evapotranspiration using artificial neural network. Journal of Irrigation and Drainage Engineering, 128(4): 224-233.

LEANDRO RF. 2004. A new technique to TEC regional modeling using a neural network. In: Proceedings of the ION GNSS, Califórnia, Long Beach, 2004. Artigos, p. 1-9.

MAIA TCB. 2003. Utilização de redes neurais artificiais na determinação de modelos geoidais. Escola de Engenharia de São Carlos - Universidade de São Paulo, Tese de Doutorado, 130 p.

MAO K \& SHI JA. 2008. Neural Network Technique for Separating Land Surface Emissivity and Temperature from ASTER Imagery. IEEE Transactions on Geoscience and Remote Sensing, 46(1): 200-208.

MIRANDA FA, DE FREITAS SRC \& FAGGION PL. 2007. Integração e interpolação de dados de anomalias ar livre utilizando-se a técnica de RNA e krigagem. In: Simpósio Brasileiro de Geomática, 2., Presidente Prudente/SP. Anais... Presidente Prudente, UNESP, 2007. Artigos, p. 69-76. CD-ROM. ISBN 1981-6251

MORITZ H. 1980. Advanced Physical Geodesy. Abacus Press, Wichmann, $500 \mathrm{p}$.

MÜLLER M \& FILL HD. 2003. Redes Neurais aplicadas na propagação de vazões. In: Simpósio Brasileiro de Recursos Hídricos, 15., Curitiba. Anais..., Curitiba: ABRH, 2003. Artigos, p. 1-15. CD-ROM, Online. Disponível em: <http://www.lactec.org.br/OInstituto/downloads/Biblioteca/2003/065_2003.pdf >. Acesso em: 11 set. 2008.

PARASURAMAN K, ELSHORBAGY A \& SI BC. 2007. Estimating saturated hydraulic conductivity using genetic programming. Soil. Sci. Soc. Am. J., 71(6): 1676-1684.

PAVLIS NK, HOLMES SA, KENYON SC \& FACTOR JK. 2008. An Earth Gravitational Model to Degree 2160: EGM2008. In: 2008 General Assembly of the European Geosciences Union, April 13-18, 2008, Viena.
Disponível em: <http://earth-info.nga.mil/GandG/wgs84/gravitymod/ egm2008/index.html> . Acesso em: 10 nov. 2009.

REINKE M, VERONEZ MR, THUM AB, DE SOUZA GC \& SEGANTINE PCL. 2007. Determinação da superfície geoidal através de redes neurais artificiais. GAEA, 3(1): 27-36.

SCHWARZ KP, SIDERIS MG \& FORSBERG R. 1989. The Use of FFT Techniques in Physical Geodesy. Geophysics Journal International, 110(1): 485-514

SEAGER J, COLLIER P \& KIRBY J. 1999. Modelling Geoid Undulations with an Artificial Neural Network. IEEE, 5(1): 3332-3335.

SILVA ANR, RAMOS RAR, SOUZA LCL, RODRIGUES DS \& MENDES JFG. 2004. SIG - Uma plataforma para introdução de técnicas emergentes no planejamento urbano, regional e de transportes. São Carlos: Editora da EESC/USP, 2004, 221 p.

SIRIPITAYANANON P, CHEN HC \& HART BS. 2001. A New Technique for Lithofacies Prediction: Back-Propagation Neural Network. In: Annual ACM Southeast Conference, 39., Proceedings of Athens - Georgia. ACM, 2001. Artigos, p. 1-8. Online. Disponível em: <http:// webster.cs.uga.edu/ jam/acm-se/review/referee/sirip001.pdf >. Acesso em: 11 set. 2008

SOUZA SF. 2002. Contribuição do GPS para o Aprimoramento do Geóide no Estado de São Paulo. Tese de Doutorado, IAG/USP, São Paulo, 240 p.

TIERRA A \& DE FREITAS SRC. 2002. Predicting Free-Air Gravity Anomaly Using Artificial Neural Network. International Association of Geodesy Symposia: Vertical Reference Systems. Springer, 124: 215-218.

VERONEZ MR, THUM AB, LUZ AS \& DA SILVA DR. 2006. Artificial Neural Network applied in the determination of Soil Surface Temperature-SST. In: International Symposium Accuracy Assessment in Natural Resources and Environmental Sciences, (Accuracy 2006), 7., Lisboa-Portugal. Anais... Lisboa, IGP, 2006. Artigos, p. 889-898. Impresso. ISBN 972886-727-I

YANG CC, PRASHER SO, MEHUYS GR \& PATNI NK. 1997. Application of artificial neural networks for simulation of soil temperature. Transactions of the ASAE, 40(3): 649-656.

YANG Y, APLIN AC \& LARTER SR. 2004. Quantitative assessment of mudstone lithology using geophysical wireline logs and artificial neural networks. Petroleum Geoscience, 10(2): 141-151.

ZACHARIAS S \& WESSOLEK G. 2007. Excluding organic matter content from pedotransfer predictors of soil water retention. Soil. Sci. Soc. Am. J., 71(1): 43-50.

ZANETTI SS, SOUSA EF, DE CARVALHO DF \& BERNARDO S. 2008. Estimação da evapotranspiração de referência no Estado do Rio de Janeiro usando redes neurais artificiais. Revista Brasileira de Engenharia Agrícola e Ambiental, 12(2): 174-180. 


\section{NOTAS SOBRE OS AUTORES}

Maurício Roberto Veronez. Possui graduação em Engenharia de Agrimensura pela Universidade Federal de Viçosa (UFV/MG) e doutorado em Engenharia Civil, área de Mensuração pela Escola de Engenharia de São Carlos (EESC/USP). Atualmente é professor adjunto I no Programa de Pós-Graduação em Geologia da Universidade do Vale do Rio dos Sinos (UNISINOS/RS) lecionando as disciplinas Cartografia Digital e Redes Neurais Artificiais.

Sérgio Florêncio de Souza. Possui graduação em Engenharia Cartográfica pela Universidade Estadual Paulista Júlio de Mesquita Filho (1994), mestrado em Sensoriamento Remoto pela Universidade Federal do Rio Grande do Sul (1997) e doutorado em Geofísica pela Universidade de São Paulo (2002).

Marcelo Tomio Matsuoka. Possui graduação em Engenharia Cartográfica e doutorado em Ciências Cartográficas pela Universidade Estadual Paulista. Atualmente é professor adjunto I no Departamento de Geodésia do Instituto de Geociências da Universidade Federal do Rio Grande do Sul. Tem lecionado disciplinas para o curso de Engenharia Cartográfica (Ajustamento de Observações, Geodésia Espacial, Levantamentos).

Alessandro Ott Reinhardt. Possui graduação em Sistemas de Informação pela Universidade do Vale do Rio dos Sinos (UNISINOS/RS). Atualmente é analista de campo da empresa Navteq do Brasil, onde desempenha atividades de geoprocessamento aplicado a sistemas de navegação veicular. 